\title{
Targeting Cancer Through NF-KB Pathway with Selected Natural Products ( $\beta$-Elemene, Puerarin and Gypenosides)
}

\author{
Bashir Ahmad ${ }^{2 *}$, Pengyu Su${ }^{1}$, Syed Rafiq Hussain Shah ${ }^{2}$, Shah Zeb Khan ${ }^{5}$, Badshah Hussain , \\ Fazal Wahid ${ }^{3}$, Hayat Ullah ${ }^{3}$, Shahab Uddin'3 , Manzoor Ahmad ${ }^{6}$ and Nasiha Begum ${ }^{3}$ \\ ${ }^{1}$ Institute of Cancer Stem Cell \& The Second Affiliated Hospital, Dalian Medical University, China \\ ${ }^{2}$ Department of Biology, The University of Haripur, I. R. Pakistan \\ ${ }^{3}$ Department of Botany, University of Malakand Chakdara, Pakistan \\ ${ }^{4}$ Department of Botany, Hazara University, Mansehra, Pakistan. \\ ${ }^{5}$ Department of Botany, Shaheed Benazir Bhuto University Sheringal, Pakistan \\ ${ }^{6}$ Department of Chemistry, University of Malakand Chakdara, Pakistan
}

*Corresponding author: Bashir Ahmad, Department of Biology, The University of Haripur, I. R. Pakistan.

To Cite This Article: Bashir A, Pengyu S, Syed Rafiq Hussain S, Shah Zeb K, Badshah H, et al., Targeting Cancer Through NF-KB Pathway with Selected Natural Products ( $\beta$-Elemene, Puerarin and Gypenosides). 2020 - 10(1). AJBSR.MS.ID.001472. D0I: 10.34297/AJBSR.2020.10.001472.

Received: 眥 May 28, 2020; Published: 眥 August 25, 2020

\begin{abstract}
Cancer is the second leading cause of mortality around the world; therefore, its immediate treatment is very necessary. Natural products (NPs) are considered more effective and less toxic among all therapies. The vital sources of these natural products are medicinal plants. Various studies have reported that the NPs cure cancer through modulation of the NF-kB pathway. Among the NPs, $\beta$-Elemene (ELE), Puerarin (Pue) and Gypenosides (Pue) possess potent anti-tumor effect via regulation of NF-kB pathway; therefore we summarize the available studies to provide a baseline for further research on these NPs.
\end{abstract}

Keywords: Cancer, Natural products, $\beta$-Elemene, Puerarin, Gypenosides

\section{Introduction}

Cancer is the second leading cause of mortality around the world, therefore, its immediate treatment is very necessary [14]. Natural products (NPs) are considered more effective and less toxic among all therapies $[1,3,4]$. The vital source of these natural products is medicinal plants $[5,6]$. NPs cure cancer via modulation of different molecular pathways, including NF-kB, MEK-ERK, autophagy, PI3K/AKT/mTOR, oxidative stress, inflammation and apoptosis [5]. Sesquiterpene lactones (SLs) are a group of NPs belong to C15 terpenoids group. These SLs possesses a variety of pharmacological and biological activities including anti-cancer and anti-inflammatory [6].
In SLs, ELE possess potent anticancer effect against different cancers [7]. The source of ELE is Rhizoma Zedoaire, which I dry rhizome of Curcuma khangsiensis, Curcuma wenyujin and Curcuma phaeocaulis [1]. The Chinese ministry of health has been approved ELE for the treatment of cancer [1]. ELE induces apoptosis through different mechanisms, including NF-kB pathway. Next, Puerarin (Pue) is also an NP derive from Pueraria lobata (Wild) ohwi, Pueraria tuberosa (Wild) and Pueraria thomsonii Benthi [5] and approved by the Chinese ministry of health for the treatment of different diseases [5]. 
The NPs Gypenosides (Gyp) belong to a triterpine saponins group which are derived from Gynostema penthaphyllum (GpM), having an anticancer effect both in vivo and in vitro as well used in different clinical trials [8]. Gyp has been used for the treatment of a number of diseases in China, including hyper-lipoprotenemia [9], cardiovascular diseases [10], and hepatitis [11]. Furthermore, in a number of cancer cell lines including, oral cancer SAS cells, [12] SW620, 2, [13] and cervical epidermoid carcinoma cells have been reported [14]. Gyp inhibits the migration, invasion, metastasis, proliferation and induces apoptosis in a variety of cancers, including lung, hepatocellular, oral, colorectal and leukemic cancer through different mechanisms including NF-kB pathway 2.

\section{NF-kB pathway and Cancer}

NF-kB (Nuclear Factor Kapbba B) is a transcription factor complex having homo and heterodimers of five members of a Reticuloenotheliosis oncogene cellular-homolog (Rel) family, including RelB, RelA (p65), c-Rel, NF-kB2 (p52/p100) and NF$\mathrm{kB1}$ (p50/p105) [15]. The functions of NF-kB is dysregulated in tumerogenesis [16]. In different cancers, including prostate, breast, pancreas, liver, colon, lymphoma, leukemia and ovarian cancers the NF-kB has been reported inactive state [17-19]. The DNA damage in lead to activation of NF-kB due to which NF-kB targeted genes are becomes activated, such are cyclooxygenase-2 (COX-2) [20] and iNOS (inducible nitric oxide synthase) [21]. Next, the TNF $\alpha$ binding to TNFR causes homotrimerization of the adopter and receptor proteins, causes cell survival and proliferation through enhancing the expression of NF-kB and activator protein 1 target genes, including vascular cell adhesion molecule 1(VCAM 1) [22-24]. Furthermore, the active NF-kB cause the activation of chemokines and its related receptors such are C-X-C chemokine receptor-4 (CXCR-4) and CCR-7 [25] which are involved in target organs [22]. These genes are involved in the anti-apoptosis and pro-survival. It is very obvious that the NF-kB is a candidate for therapeutic resistance in a variety of cancers. NPs possess potent therapeutic activity against different cancers through regulation of NF-kB pathway $[6,26]$.

Targeting Cancer through NF-kB pathway with NPs ( $\beta$-Elemene, Puerarin and Gypenosides). One of the most important and potent NP ELE shows its anticancer activities against different type cancer cells, including RPMI-8226, SGC7901/ADM and HL-60 through modulation of NF-kB pathways. In NF-kB pathway, ELE inhibit NF-kB p65, COX-2, PGE2 and lead to inhibition of cell proliferation [27-29] as depicted in Figure 1. Another NP Pue has a potent effect against different cancer cell lines including, lipopolysaccharide induced THP1 [30], Z138 [31], T24 [32], MCF-7 [33], MCF-7/ Adriamycin (MCF-7/adr) [34], and MDA-MB-231 [33].

Figure 1: NPs (ELE, Pue and Gyp) and NF-kN pathway in different cancers. In NF-kB pathway, ELE inhibit NF-kB p65, COX-2, PGE2 and lead to inhibition of cell proliferation. Pue downregulate the expression of TNF- $\alpha$ and IL- 6 and Pue inhibits the expression of inflammatory factors TNF- $\alpha$ and IL-6 and reduce the activation of NF-kB through downregulation of p-IkBa/lkBa, IkkappaB and p65 while increasing the expression of miR16 Next, Pue inhibit the nuclear translocation of NF-kB [33] which result in downregulation of COX-2, MMP-2,9, CXCR-4, CCR-7, VCAM, and ICAM, both at mRNA and protein level. Gyp downregulate the SOS, RAS, uPA and FAK, which further reduces the expression of AKT, NF-kB, iNOS and COX-2 while activating p53. Furthermore, they inhibit MMP-2,7,9 which result in inhibition of cell migration, invasion and metastasis. 
In these cells, Pue negates adhesion [33], inhibit migration [33], invasion [33] and proliferation [31] through modulation of NF-kB pathway [30,31,33-35]. Furthermore, Pue downregulate the expression of TNF- $\alpha$ and IL- 6 and Pue inhibits the expression of inflammatory factors TNF- $\alpha$ and IL-6 [33] and reduce the activation of NF-kB through downregulation of $\mathrm{p}-\mathrm{IkB} \alpha / \operatorname{IkB} \alpha[30$, $33,34]$ IkkappaB $[33,34]$ and p65 while increasing the expression of miR16 [32]. Next, Pue inhibit the nuclear translocation of NF$\mathrm{kB}$ [35] which result in downregulation of COX-2, MMP-2,9, CXCR4, CCR-7, VCAM, and ICAM, both at mRNA and protein level [33]. Natural Gyp possesses anticancer effect against SAS and SCC-4 cells through NF-kB pathway via downregulation of SOS, RAS, uPA and FAK, which further reduces the expression of AKT, NF-kB, iNOS and COX-2 while activating p53. Furthermore, they inhibit MMP-2,7,9 which result in inhibition of cell migration, invasion and metastasis $[12,36]$ as shown in Figure 1.

\section{Conclusions}

NPs play pivotal role in cancer therapy. Among these NPs, the ELE, Pue and Gyp possesses the anti-tumor effect through modulation of NF-kB pathways. Further, the mechanisms have been summarized in Figure 1.

\section{Conflict of Interest}

The authors declare no conflict of interest.

\section{Consent for Publication}

All authors agree to be published.

\section{References}

1. AB Pengyu S, Lijuan Z (2018) Natural $\beta$-Elemene: Advances in Targeting Cancer Through Different Molecular Pathways. North American Journal of Acedamic Research 1(4): 27

2. B Ahmad, S Khan, G Nabi, Y Gamallat, P Su, et al. (2019) Natural gypenosides: targeting cancer through different molecular pathways. Cancer management and Research 11: 2287-2297.

3. P Su, B Ahmad, M Ahmad, J Khan, F Wahid, et al. (2020) Targeting Cance through PI3K/AKT/mTOR Pathway with Selected Natural Products ( $\beta$-Elemene, Puerarin and Gypenosides). American Journal of Biomedical Sciences and Research 8(4): 335-338.

4. BA Pengyu Su, Hayat Ullah, Badshah Hussain, Shah Zeb Khan, Mohammad, et al. (2020) Targeting Cancer Through Autophagy With $\beta$-Elemene and Puerarin. American Journal of Biomedical Sciences and Research 8(1): 51-53.

5. B Ahmad, S Khan, Y Liu, M Xue, G Nabi, et al. (2020) Molecular Mechanisms of Anticancer Activities of Puerarin. Cancer management and research 12: 79-90.

6. S Jalal, B Ahmad, T Zhang, L Guo, L Huang, et al. (2020) SANTAMARINE: Mechanistic Studies on Multiple Diseases. Chemical Biology Drug Design 95(4): 427-434.

7. Z Jiang, JA Jacob, DS Loganathachetti, P Nainangu, B Chen (2017) Beta-Elemene: Mechanistic Studies on Cancer Cell Interaction and Its Chemosensitization Effect. Frontiers in pharmacology 8: 105.
8. Y Li, W Lin, J Huang, Y Xie, W Ma, et al. (2016) Anti-cancer effects of Gynostemma pentaphyllum (Thunb.) Makino (Jiaogulan) Chinese medicine 11(1): 43.

9. YH Yang, J Yang, QH Jiang (2013) Hypolipidemic effect of gypenosides in experimentally induced hypercholesterolemic rats. Lipids Health Disease 12: 154.

10. H Yu, Q Guan, L Guo, H Zhang, X Pang, et al. (2016) Gypenosides alleviate myocardial ischemia-reperfusion injury via attenuation of oxidative stress and preservation of mitochondrial function in rat heart. Cell stress \& chaperones 21(3): 429-437.

11. MH Chen, QF Wang, LG Chen, JJ Shee, JC Chen, et al. (2009) The inhibitory effect of Gynostemma pentaphyllum on MCP-1 and type I procollagen expression in rat hepatic stellate cells. Journal of ethnopharmacology 126(1): 42-49.

12. KW Lu, JC Chen, TY Lai, JS Yang, SW Weng, et al. (2011) Gypenosides inhibits migration and invasion of human oral cancer SAS cells through the inhibition of matrix metalloproteinase-2 -9 and urokinaseplasminogen by ERK1/2 and NF-kappa B signaling pathways. Human \& experimental toxicology 30(5): 406-15.

13. H Yan, X Wang, Y Wang, P Wang, Y Xiao (2014) Antiproliferation and anti-migration induced by gypenosides in human colon cancer SW620 and esophageal cancer Eca-109 cells. Hum Exp Toxicol 33(5): 522-33.

14. TH Chiu, JC Chen, JG Chung (2003) N-acetyltransferase is involved in gypenosides-induced $\mathrm{N}$-acetylation of 2-aminofluorene and DNA adduct formation in human cervix epidermoid carcinoma cells (Ca Ski). In vivo 17(3): 281-288.

15. R Sen, D Baltimore (1986) Multiple nuclear factors interact with the immunoglobulin enhancer sequences. Cell 46(5): 705-716.

16. ND Perkins (2007) Integrating cell-signalling pathways with NF-kappaB and IKK function. Nature reviews Molecular cell biology 8(1): 49-62.

17. MC Arkan, FR Greten (2011) IKK- and NF-kappaB-mediated functions in carcinogenesis, Curr Top Microbiol Immunol 349: 159-169.

18. DS Basseres, AS Baldwin (2006) Nuclear factor-kappaB and inhibitor of kappaB kinase pathways in oncogenic initiation and progression. Oncogene 25(51): 6817-6830.

19. S Prasad, J Ravindran, BB Aggarwal (2010) NF-kappaB and cancer: how intimate is this relationship. Mol Cell Biochem 336(1-2): 25-37.

20. K Yamamoto, T Arakawa, N Ueda, S Yamamoto (1995) Transcriptional roles of nuclear factor kappa B and nuclear factor-interleukin-6 in the tumor necrosis factor alpha-dependent induction of cyclooxygenase-2 in MC3T3-E1 cells. J Biol Chem 270(52): 31315-31320.

21. JH Park, YJ Jeong, HK Won, SY Choi, JH Park, et al. (2014) Activation of TOPK by lipopolysaccharide promotes induction of inducible nitric oxide synthase through NF-kappaB activity in leukemia cells. Cellular signalling 26(5): 849-856.

22. A Müller, B Homey, H Soto, N Ge, D Catron, et al. (2001) Involvement of chemokine receptors in breast cancer metastasis. Nature $410(6824)$ : 50-56.

23. GD Kalliolias, LB Ivashkiv (2016) TNF biology, pathogenic mechanisms and emerging therapeutic strategies. Nature reviews rheumatology 12(1): 49-62.

24. D Brenner, H Blaser, TW Mak (2015) Regulation of tumour necrosis factor signalling: live or let die. Nat Rev Immunol 15(6): 362-374.

25. UE Hopken, HD Foss, D Meyer, M Hinz, K Leder, et al. (2002) Up-regulation of the chemokine receptor CCR7 in classical but not in lymphocytepredominant Hodgkin disease correlates with distinct dissemination of neoplastic cells in lymphoid organs. Blood 99(4): 1109-11016. 
26. T Nabekura, T Hiroi, T Kawasaki, Y Uwai (2015) Effects of natural nuclear factor-kappa B inhibitors on anticancer drug efflux transporter human P-glycoprotein. Biomed Pharmacother 70: 140-145.

27. CP Zheng, XM Tong, HP Yao, J Yang, J Xu, et al. (2009) [beta-elemene enhances aclarubicin-induced apoptotic effect in HL-60 cells and its mechanism]. Zhonghua Xue Ye Xue Za Zhi 30(12): 821-824.

28. TH Fu, JY Li, YY Jing, PJ Sun, X Bai (2013) [Effect of elemene on reversing chemoresistance to adriamycin in human stomach cancer cell line] Zhong Yao Cai 36(4): 601-603.

29. H Chen, L Shi, ZY Cheng, L Yao, YY Yang, et al. (2010) [Effects of betaelemene on proliferation and apoptosis of human multiple myeloma cell RPMI-8226]. Zhongguo Shi Yan Xue Ye Xue Za Zhi 18(2): 368-371.

30. H Zhang, Z Zhai, H Zhou, Y Li, X Li, et al. (2015) Puerarin Inhibits oxLDLInduced Macrophage Activation and Foam Cell Formation in Human THP1 Macrophage. BioMed research international 403616.

31. M Gan, X Yin (2015) Puerarin induced in mantle cell lymphoma apoptosis and its possible mechanisms involving multi-signaling pathway. Cell biochemistry and biophysics 71(1): 367-373.
32. X Liu, S Li, Y Li, B Cheng, B Tan, et al. (2018) Puerarin Inhibits Proliferation and Induces Apoptosis by Upregulation of miR-16 in Bladder Cancer Cell Line $\mathrm{T}_{24}$. Oncology research 26(8): 1227-1234.

33. X Liu, W Zhao, W Wang, S Lin, L Yang (2017) Puerarin suppresses LPSinduced breast cancer cell migration, invasion and adhesion by blockage NF-kappaB and Erk pathway. Biomedicine \& Pharmacotherapy 92: 429436.

34. TT Hien, HG Kim, EH Han, KW Kang, HG (2010) Jeong, Molecular mechanism of suppression of MDR1 by puerarin from Pueraria lobata via NF-kappaB pathway and cAMP-responsive element transcriptional activity-dependent up-regulation of AMP-activated protein kinase in breast cancer MCF-7/adr cells. Molecular nutrition \& food research 54(7): 918-28.

35. X Liu, S Li, Y Li, B Cheng, B Tan, et al. (2018) Puerarin inhibits proliferation and induces apoptosis by up-regulation of miR-16 in bladder cancer cell line T24. Oncol Res 26(8): 1227-1234.

36. KW Lu, ML Tsai, JC Chen, SC Hsu, TC Hsia, et al. (2008) Gypenosides inhibited invasion and migration of human tongue cancer SCC4 cells through down-regulation of NFkappaB and matrix metalloproteinase-9. Anticancer Res 28(2A): 1093-1099. 